\title{
LOS PRECEPTOS DE LA CIENCIA DE LA MOTRICIDAD HUMANA Y LOS PROFESIONALES DE EDUCACIÓN FÍSICA EN LA SOCIEDAD CONTEMPORÁNEA: UN ANÁLISIS DESDE LA PERSPECTIVA DE LOS PROYECTOS DEPORTIVOS SOCIALES
}

\section{THE PRECEPTS OF HUMAN MOVEMENT SCIENCE AND PHYSICAL EDUCATION PROFESSIONALS IN CONTEMPORARY SOCIETY: AN ANALYSIS FROM THE PERSPECTIVE OF PROJECTS SOCIAL AND SPORTS}

\author{
Machado Tibério ${ }^{1,2}$ \& Vargas Angelo, 2,3 \\ ${ }^{1}$ Mestre em Ciência do Movimento Humano pela Universidade Autônoma de Assunção, PY \\ ${ }^{2}$ Laboratório de Estudos da Cultura Social Urbana \\ ${ }^{3}$ Doutorado em Ciência da Motricidade Humana pela Universidade Técnica de Lisboa, PT
}

MACHADO T.\& VARGAS A. Los Preceptos de la Ciencia de la Motricidad Humana y los Profesionales de Educación Física en la Sociedad Contemporánea: Un Análisis desde la Perspectiva de los Proyectos Deportivos Sociales. Mot.Hum. 13(2); 104-109: 2012.

\section{RESUMEN}

La relación entre el Hombre y la práctica de actividad física ha obtenido fama reconocida en los últimos años. Sin embargo, la actuación del Profesional de Educación Física en la gestión de esta relación, a pesar de efectiva, tenía una carencia de conocimiento técnico y científico, un hecho que dificultó la consolidación del trabajo en la sociedad contemporánea. En esto universo emergió la Ciencia de la Motricidad Humana, un área del conocimiento científico que ha surgido con la función de proporcionar apoyo científico y técnico para los Profesionales de Educación Física, fundamentalmente al mostrar que le deporte trasciende el universo deportivo y una nueva comprensión de Hombre es posible. Vale la pena señalar que estos preceptos han logrado un éxito considerable, principalmente en la República Federativa del Brasil, que se caracterizó por la relación indiscutible entre el Hombre y la práctica de actividad física, junto con el fomento través de mecanismos legales para el crecimiento del deporte y asegurar en reconocimiento de los Profesionales de Educación Física.

Palabras Clave: Deportes; Política Social; Motricidad Humana.

\begin{abstract}
The relationship amid man and physical activity has become notorious over the years. However, the performance of the Professional Physical Education in the management of this relationship although effective, had lack of technical and scientific support, event that hampered the consolidation of this professional in contemporary society. In this universe emerged the Science of Human Movement, an area of scientific knowledge that has appeared with the task of providing scientific and technical support for Physical Education Professionals, mainly by showing that sports transcends the sports scene and a new understanding of man was needed. It is worth noting that these precepts have achieved substantial success, especially in the Federative Republic of Brazil, which was characterized by the relationship between man and undisputed physical activity, and through legal mechanisms promote sports and ensure recognition the Professional Physical Education.
\end{abstract}

Key Words: Sports; Public Policy; Human Movement. 
MACHADO T.\& VARGAS A. Los Preceptos de la Ciencia de la Motricidad Humana y los Profesionales de Educación Física en la Sociedad Contemporánea: Un Análisis desde la Perspectiva de los Proyectos Deportivos Sociales. Mot.Hum. 13(2); 104-109: 2012.

\section{INTRODUCCIÓN}

La historia humana presentó, como peculiaridad, su desarrollo a través de la actividad física. En algunas ocasiones, debido a la lucha por la sobrevivencia, en otros con el fin de prepararse para los posibles conflictos o disputas territoriales, en otras palabras, situaciones con característica guerrera, allá de la forma de la danza o recreación que se convirtió en una particularidad indiscutible y notable (1). Sin embargo, ha tenido un periodo de gran importancia histórica, y respecta a la época donde el deporte y la actividad física se asociaron con el status de belleza y sabiduría, una característica típica de la Grecia Antigua (2). No obstante, la acción efectiva del Profesional de Educación Física, regulado en la sociedad, así como la consolidación y difusión de los preceptos de la Ciencia de la Motricidad Humana en el ámbito de la Educación Física, emergió en la sociedad contemporánea, donde en el escenario brasileño demostró ser un período de valorización y fomento de los deportes. Este conjunto de conocimiento ha resaltado una nueva visión en la concepción del conocimiento de la actividad física y del universo del deporte (3).

Es imprescindible argumentar que surgen cuestionamientos en la relación entre los fundamentos de la Ciencia de la Motricidad Humana, con la forma de la actuación y la formación de los Profesionales de la Educación Física, que actualmente incluyen la visibilidad y la notoriedad por su participación en los proyectos socio deportivos.

\section{LA CIENCIA DE LA MOTRICIDAD HUMANA: UNA CONTRIBUCIÓN AL DESARROLLO DE LA EDUCACIÓN FÍSICA Y LA FORMACIÓN DE LOS PROFESIONALES}

La ciencia puede ser comprendida de acuerdo con los conceptos inferidos por Bereoff como área del conocimiento que tiene como finalidad el desarrollo de investigaciones específicas, métodos y procedimientos adecuados que tienen por objeto proporcionar el desarrollo, la adquisición y la exhibición de conocimientos a través de la demostración y presentación de los resultados (4).
Sin embargo, los cuestionamientos siguen en la Educación Física, principalmente por la terminología Educación Física. Para Sérgio este termino no refleja la realidad, siendo reduccionista. Además, esta área del conocimiento en el punto de vista de este autor, constituye la ciencia inicial para la Educación de la Motricidad, que basado en los preceptos de la Ciencia de la Motricidad Humana es una forma de conciencia que pretende negar el positivismo, presentando cientificidad a una área que no tiene (5). Para Feitosa:

"La ciencia de la motricidad humana, es um esfuerzo para darle cienticidad a una profesión que no la tiene; y simultaneamente una lucha para que se conceda espacio institucional al desarrollo de esta especialidad cientifica"(6).

De acuerdo con Morin la cientificidad es determinante para cualquier ciencia o área del conocimiento. El autor señala que: "el proposito de ciência no es reflejar la realidade, pero traducir la teoria de mutable o refutable." (7). Este tipo de pensamiento, razonamiento $\mathrm{o}$ argumento contribuye a favor y el apoyo de los preceptos de Sérgio, no respecta el desarrollo científico de la Educación Física y sus Profesionales (3). La ciencia como una estrategia para la producción y consolidación del conocimiento, tiene la responsabilidad de buscar la aproximación de la verdad (8), verdad que no es absoluta, porque es inalcanzable, en otras palabras, es imposible (9). Profundizando las reflexiones de este tema en especial en el campo semántico de la Educación Física, todo el propósito de la busqueda del progreso científico, tiene razón debido a la carencia evidente en la Educación Física convencional, que puede verse afectada aún más debido a la ocurrencia del fenómeno conocido como ciencia normal. En el sentido de Kuhn, la ciencia normal fue definida como:

"la actividad del professional no revolucionario, o mejor dicho, no muy crítico: es el erudito de la ciencia que acepta el dogma dominante del día: no quieren oponerse a ella, y que sólo acepta una nueva teoría revolucionaria, cuando casi todo el mundo está listo para aceptarla cuando pasa a estar de moda, como una 
MACHADO T.\& VARGAS A. Los Preceptos de la Ciencia de la Motricidad Humana y los Profesionales de Educación Física en la Sociedad Contemporánea: Un Análisis desde la Perspectiva de los Proyectos Deportivos Sociales. Mot.Hum. 13(2); 104-109: 2012.

candidatura antecipadamente victoriosa a la que todos se adhieren" (10).

En el caso particular de la Educación Física, tal como presentó Sérgio en su proposición para la Ciencia de la Motricidad Humana (3), este fenómeno no fue asimilado por todos los profesionales. Con el fin de una mejor sustentación teórica, Sérgio propuso la construcción de un estatuto epistemológico para la Educación Física. La epistemología es un análisis, una reflexión de toda la producción existente (11). Este hecho corrobora con los objetivos propuestos por Manuel Sérgio a través de la Motricidad Humana, que es la realización de cuestionamientos a los principios y conocimientos imperantes de la Educación Física (11).

Es posible asegurar que desde esta nueva perspectiva, que permitió una visión amplia, llego a ser posible oponerse a la educación física convencional, que utilizaba como fundamentación la teoría cartesiana, que fue creada por el filósofo René Descartes y propuso en sus postulados filosóficos la división del Hombre en dos partes bien diferenciadas, res cogitans (mente) y res extensa (materia) (3). En los preceptos de la Ciencia de la Motricidad Humana, Sérgio contrario el cartesianismo, presentando una visión amplia e ininterrumpida del Hombre, siendo un ser único e indivisible, inseparable, no aceptando las muchas técnicas existentes, que son carentes de objetividad y cientificidad, además de no favorecer el desarrollo del Hombre como un ser $(3,11)$.

El Hombre tiene peculiaridades que dificultan su entendimiento y comprensión. Cuando está presente en su medio social, es decir, ambiente de interacción social, un acontecimiento frecuente en la vida humana, sus características aumentan sustancialmente de complejidad, debido principalmente a las interacciones sociales, que son actos de la vida que pueden haber sido heredadas en nuestro proceso evolutivo (12). Marx y Engels han reportado en sus postulados filosóficos la importancia de esta relación, donde el Hombre sufre las intervenciones del ambiente social en el que se inserta o en el que tiene significativo contacto (13).
Sin embargo, como la Ciencia de la Motricidad Humana surge en esta historia, o en la busca de la comprensión humana, en sus fundamentos, Sérgio presentó oposición a la teoría cartesiana que conducía la Educación Física que estaba en vigor, basado en el principio que cada humano tiene características diferentes (3). En el presente sentido, Ortega y Gasset presentó una célebre reflexión, que demuestra que no somos todos iguales: "Yo soy yo y mis circunstancias, y si las salvo a ellas me salvo yo" (14). En este universo la Ciencia de la Motricidad Humana afirma la necesidad de recordar que el Hombre es indivisible, único, o uno, acción que expone un raciocinio diferente de lo idealizado por el cartesianismo (3).

\section{REGLAMENTACIÓN Y EL DESARROLLO DE LA EDUCACIÓN FÍSICA EN LA SOCIEDAD CONTEMPORÁNEA}

La práctica de la actividad física ha acompañado la humanidad desde sus origines, sin embargo, la actuación del Profesional de la Educación Física, especialmente en la sociedad brasileña, país que se guió por los preceptos diseminados por Manuel Sérgio en el campo semántico de la Ciencia de la Motricidad Humana, que solo obtiene su reglamento después de la promulgación de la Ley no 9696/98, el 1 de septiembre de 1998 (15). Esta fecha constituye una fecha de referencia para los profesionales brasileños. A partir entonces, pasó a ser responsabilidad del Consejo Regional de la Educación Física - CREF y el Consejo Federal de la Educación Física - CONFEF, la supervisión de la práctica profesional y los cursos de formación para los profesionales de esta área del conocimiento (15).

El Artículo 217 de la Constitución de la República Federativa del Brasil, publicada en el 5 de octubre de 1988, prevé la diseminación del deporte en tres diferentes dimensiones sociales: deportes educación, deporte participación y deporte rendimiento (16). El deporte educación presentó como característica principal la práctica deportiva basada en la diseminación de los principios educativos como la participación y la integración de los participantes. No obstante, el deporte participación tiene como objetivo el desarrollo del deporte como una ocupación para el tiempo libre, además de representar una alternativa de 
MACHADO T.\& VARGAS A. Los Preceptos de la Ciencia de la Motricidad Humana y los Profesionales de Educación Física en la Sociedad Contemporánea: Un Análisis desde la Perspectiva de los Proyectos Deportivos Sociales. Mot.Hum. 13(2); 104-109: 2012.

entretenimiento y recreación, acontecimiento que significan una forma efectiva de oposición al ocio. Por último el deporte rendimiento, que hoy se destaca con gran visibilidad en la sociedad y tiene por objetivo el desarrollo del deporte para la competición o las disputas, siguiendo las normas y las reglas de las instituciones y organizaciones deportivas (17).

Aunque diversos objetivos pueden lograrse en el desarrollo de la práctica deportiva, esta versatilidad ha contribuido para el desenvolvimiento y consolidación del deporte en la sociedad moderna, como consecuencia de los profesionales que trabajan en esta área. En este campo semántico, lo sesgo de la salud social ha logrado gran importancia, pero los dogmas imperantes entre la población, emergieron como una de las razones principales para el crecimiento de otras vertientes, tales como la búsqueda de la belleza física.

El culto al cuerpo, la procura por el ideal hermoso de la sociedad, floreció como uno de los principales campos de acción, teniendo su origen en la historia de la humanidad en el siglo XV y XVI. Autores como Castilho infieren sobre el tema, que debe interpretarse en el contexto de la busqueda de una manera de aceptación en un grupo social (18), como presentó el filósofo Michel Foucault, que afirmó en su postulado que los seres humanos ansían estos objetivos, fundamentalmente como el resultado de la búsqueda de la aceptación social (19). Debe tenerse en cuenta, la parte de responsabilidad de los Profesionales de la Educación Física en este asunto, que debido la incapacidad o limitación, como comentó Manuel Sérgio, no habían aspirado al desarrollo de otros aspectos en los seres humanos, acontecimiento que favoreció a su estancamiento $\mathrm{y}$ al apego a los dogmas $\mathrm{y}$ paradigmas existentes, promoviendo a "la atrofia del deporte", ya que el potencial no está siendo utilizado o desenvuelto, además de ser un objetivo de magnitud individual y relevancia discutible para la sociedad en el escenario de la comunidad (5).

EDUCACIÓN FÍSICA $\quad$ Y
PROFESIONALES ENS
CONTEMPORÁNEA: ATENCIÓN DE LAS
NECESIDADES CONTEXTUALES

La sociedad contemporánea expresa la característica de fomentar el crecimiento de los deportes, así como el aumento de los problemas sociales en su contexto, promoviendo así el desenvolvimiento del deporte y de la actuación de los Profesionales de Educación Física en una nueva perspectiva. Se infiere que esta nueva forma de actuación de los profesionales, ha demandado mayor participación, debido a la importancia y a las particularidades existentes en su trabajo, que tuvo como finalidad ayudar en las necesidades sociales de la población, teniendo el deporte como estrategia fundamental (20).

En este período, una serie de reflexiones y propuestas emanan del universo de la Educación Física, principalmente en la forma de actuación de los profesionales, un hecho que llevó a discusiones profundas sobre la manera que estaban siendo conducidos los cursos de formación. Para pensadores como Tojal, el Profesional de la Educación Física, demostró en su trabajo la falta de conocimiento, siendo conocido por un profesional con perfil educador, no obstante, que presentó la falta de entendimiento de diversos fundamentos inherentes y necesarias para su desempeño. Por lo tanto, el surgimiento del curso de Bachillerato en Educación Física, tiene el objetivo de satisfacer las carencias y profundar en el conocimiento utilizado en el curso de formación, promoviendo una división en el proceso de formación que restringió la licenciatura al contexto de la escuela (21).

En lo que respecta a la práctica profesional en la sociedad contemporánea, como se discutió hasta ahora, es posible decir que los Profesionales de Educación Física se destacaran con mayor aceptación en la población, en las políticas deportivas con finalidad social y que creció en la sociedad brasileña junto con los problemas sociales (20). El Profesional de Educación Física que actúa en este escenario, requiere utilizar toda la ingeniosidad presente en el deporte, con la función de alcanzar los objetivos asignados, que supere el terreno deportivo y exija un conocimiento y una actuación profesional como hasta la fecha rara vez se informó. Cabe señalar que el deporte como fenómeno social consolidado y con situaciones únicas en las cuestiones y tramas sociales, junto con las nuevas fundamentaciones extendidas en el universo de la Educación Física, 
MACHADO T.\& VARGAS A. Los Preceptos de la Ciencia de la Motricidad Humana y los Profesionales de Educación Física en la Sociedad Contemporánea: Un Análisis desde la Perspectiva de los Proyectos Deportivos Sociales. Mot.Hum. 13(2); 104-109: 2012.

encaminó este profesional con poco apoyo científico y técnico para el desenvolvimiento, convirtiéndolo en responsable de la expresión y propagación de los deportes en la sociedad contemporánea, como los proyectos deportivos con fines sociales (22).

\section{OBSERVACIONES FINALES}

Los argumentos presentados favorecen la comprensión de que la Ciencia de la Motricidad Humana, en los ideales de Manuel Sérgio tuvo como objetivo la transformación del campo de la Educación Física, con la función de promover el desarrollo de un área tradicional del conocimiento humano, sin embargo, que carece de estudios e investigaciones, presentando sus consideraciones y razonamientos basados en la vivencia empírica, las experiencias particulares o de observaciones superficiales, que no contemplan o no consideraban el contexto de inserción $\mathrm{o}$ complejidad que presenta el individuo (3).

Estos preceptos se extendieron, especialmente en la República Federativa del Brasil, país que así como otros, muestra en su historia una fuerte relación con el deporte y la práctica deportiva, sin embargo la actuación oficial de los Profesionales de la Educación Física es algo nuevo en la sociedad. Hasta los días actuales las formas y áreas de participación de este profesional no se han definido con certeza, un hecho que ayuda para el desarrollo de lagunas en el conocimiento y dudas entre los actuantes en el área, sobre todo por la falta de producción e investigación científica.

En el caso específico de los proyectos deportivos con fines sociales, proyectos deportivos sociales, que son iniciativas que han surgido con la finalidad de ayudar a la población, que han sufrido por años con la atención inadecuada o ninguna. Los argumentos presentados permiten concluir que el deporte se ha convertido en efector para el logro de los objetivos, en otras palabras, los profesionales son los responsables, los conductores o los mediadores, que a lo largo de los años se halla contaminados por la agudeza y la versatilidad del deporte, siendo necesario adoptar una postura favorable para el logro de los objetivos, que proporcionan la interacción entre las diferentes dimensiones sociales del deporte, que se introdujeron en el Artículo 217 de la
Constitución de la República Federativa del Brasil en 1988 (16).

Por lo tanto, fue posible concluir que los proyectos sociales deportivos, así como los profesionales que trabajan en este tipo de iniciativa, son un punto importante, debido a la significancia del trabajo en este tipo de iniciativa, que alcanzo el respecto y la admiración de la sociedad tal como presentó Zaluar (20). Otro aspecto que debe ser presentado, se refiere a la producción científica en esta área del conocimiento, donde los investigadores como Sérgio, Zaluar, Tubino, Gonçalves, Lamartine, Vargas y otros $(20,22)$, realizaran diversos estudios, con el fin de comprender los proyectos deportivos, la actuación y el desempeño del Profesional de la Educación Física, además de las características contextuales de las localidades de inserción de los proyectos. La interacción de diversos estudiosos, compite para proporcionar una profunda investigación y entendimiento del universo constituido por los proyectos deportivos sociales, que pueden haber contribuido para el desarrollo de este profesional, que hasta este momento en la historia humana fue clasificado como incapaz, carente de conocimiento, de no goza de respeto y reconocimiento en la sociedad, como informó Manuel Sérgio en los preceptos epistemológicos de la Ciencia de la Motricidad Humana.

\section{REFERENCIA BIBLIOGRÁFICAS}

1. Rubio K. Do olimpo ao pós-olimpismo: elementos para uma reflexão sobre o esporte atual. Revista Paulista de Educação Física. 2002;16(2):130-43.

2. Sigoli MA, Junior DR. A história do uso político do esporte. Revista Brasileira de Ciência e Movimento. 2004;12(2):111-19.

3. Sérgio M. Motricidade Humana: um objeto de estudo. Motrivivência. 1988;1:8-14.

4. Bereoff OS. Epistemologia da motricidade humana. Integração. 2007; 48(13):55-59.

5. Sérgio M. Motricidade Humana: contribuições para um paradigma emergente. Lisboa: Instituto Piaget. 1994.99. 
MACHADO T.\& VARGAS A. Los Preceptos de la Ciencia de la Motricidad Humana y los Profesionales de Educación Física en la Sociedad Contemporánea: Un Análisis desde la Perspectiva de los Proyectos Deportivos Sociales. Mot.Hum. 13(2); 104-109: 2012.

6. Feitosa AM. Contribuições de Thomas Khun para uma Epistemologia da Motricidade Humana. Lisboa: Instituto Piaget.1993.32.

7. Morin E. Ciência com consciência. Lisboa: EuropaAmérica, 1984.30.

8. Feitosa AM. Contribuições de Thomas Khun para uma Epistemologia da Motricidade Humana. Lisboa: Instituto Piaget.1993.54.

9. Moura Z. A Filosofia do Erro em Karl Popper. Portugal: Regra do Jogo. 1977/78.

10. Feitosa AM. Contribuições de Thomas Khun para uma Epistemologia da Motricidade Humana. Lisboa: Instituto Piaget.1993.203.

11. Sérgio M. Para uma Epistemologia da Motricidade Humana. Portugal: Compendium. 1987.

12. Morris D. O Macaco Nu. Rio de Janeiro: Record.1967. 182.

13. Marx K, Engels F. Manifesto Comunista. Brasil: Rocket. 1999.

14. Ortega y Gasset, J. Meditaciones sobre la literatura y el arte. Madrid: Clásicos Castalia. 1987. 394.
15. Brasil (1998). Lei 9696. Regulamentação da Profissão de Educação Física e criação dos respectivos Conselho Federal e Conselhos Regionais de Educação Física. [acessado em 02 de Janeiro de 2011]. Disponível em: http://www.planalto.gov.br/ccivil_03/leis/L9696.htm

16. Brasil (1988). Constituição da República Federativa do Brasil: promulgada em 5 de outubro de 1988. [acessado em 07 de Março de 2010]. Disponível em: http://www.senado.gov.br/sf/legislacao/const/con1988/ CON1988_05.10.1988/index.htm.

17. Böhme MTS. Relações entre aptidão física, esporte e treinamento esportivo. Revista Brasileira de Ciência e Movimento. 2003 jul./set.;11(3):97-104.

18. Castilho EG. La tirania de la belleza, um problema educativo hoy. Teoría de la educación. 2002 mar.;14. 185-206.

19. Foucault M. História da sexualidade: o cuidado de si. São Paulo: Graal. 1984.

20. Zaluar A Cidadãos não vão ao paraíso. São Paulo: Escuta. 1994.

21. Tojal JBAG. Currículo de graduação em educação física: a busca de um modelo. Campinas: Edunicamp. 1989.

22. Tubino MJG. Dimensões sociais do esporte. São Paulo: Cortez. 2001.

\section{Dirigir Correspondencia a:}

Tibério Costa José Machado

tiberiojose@gmail.com

Rua Cirne Maia, 53 Bloco D, Apartamento 301 - Cachambi - Rio de Janeiro - Brasil

(21) 78452906 / (21) 25931492

\section{RECIBIDO: 18-07-2012 \\ ACEPTADO: 22-09-2012}

